\title{
Secondary hypertension accompanying high pressure chronic retention
}

\author{
R.R. Ghose and J.L. Birks \\ Singleton Hospital, Sketty, Swansea SA28QA, Wales.
}

\begin{abstract}
Summary: Two middle-aged men were referred for control of severe hypertension, presumed to be 'essential'. Late-onset enuresis, a small palpable bladder, and slight prostatic enlargement were elicited in one patient; history and examination were unremarkable in the other patient, apart from moderate prostatic enlargement. Intravenous urography revealed massive bilateral hydronephrosis with hydroureters in both patients. Surgical relief of retention was associated with post-obstructive diuresis and normalization of blood pressure, although recurrence of hypertension occurred later in one patient. These patients suffered from high pressure chronic retention, a syndrome characterized by impairment of renal function and hypertension. Surgical relief of retention was accompanied by prompt correction of hypertension, and gradual, sustained recovery of renal function.
\end{abstract}

\section{Introduction}

Chronic retention of urine may be subdivided into two groups according to urodynamic criteria: (i) high pressure, with raised intrinsic detrusor pressure during the filling phase of micturition, and (ii) low pressure, with normal pressure during filling (Abrams et al., 1978). The sequence of events in high pressure chronic retention (HPCR) consists of gradual asymptomatic bladder distension, dilatation of the lower ureters and renal pelvis, and bilateral hydronephrosis and hydroureters. Raised serum creatinine reflects an advanced stage in this process. Clinical features include lateonset enuresis, a tense, palpable bladder and hypertension. Symptoms of an obstructed outflow tract are typically absent. Intravenous urography confirms the presence of bilateral hydronephrosis with hydroureters. Untreated, the disorder inevitably progresses to renal failure (George et al., 1983). Recognition of HPCR is important in order to define a surgically treatable cause of hypertension and renal insufficiency. Observations on the effect of surgical relief of retention on these variables are reported.

\section{Patients, methods and results}

Blood pressure was measured by mercury sphygmomanometer, reading diastolic blood pressure at phase 4. Glomerular filtration rate (GFR) and effective renal plasma flow (ERPF) were measured simul-

R.R. Ghose, F.R.C.P.; J.L. Birks, Ph.D

Accepted: 29 February 1984 taneously by single-shot clearance studies of ${ }^{51} \mathrm{Cr}$ EDTA and ${ }^{125}$ I-hippuran respectively, as previously described (Morgan et al., 1977).

Renography with blood-background subtraction was performed on well-hydrated patients with a gamma camera and data processing system after the intravenous injection of ${ }^{99} \mathrm{Tc}^{\mathrm{m}}$-DTPA. The time in seconds from injection to the peak of the renogram curve ( $T$ peak), although not by itself a true physiological parameter, has been found to be a useful guide in judging individual kidney response and has a significant correlation with mean transit time (Amuasi et al., 1983). Our normal range for $\mathrm{T}$ peak is $225 \pm 42 \mathrm{~s}$ (1 s.d.).

\section{Case 1}

A 51 year old male had experienced enuresis at night and after his afternoon nap for two months, accompanied by anorexia and loss of strength. There was no hesitancy or frequency and urine volume was normal. The bladder was palpable, and tense. Blood pressure was $160 / 120 \mathrm{~mm} \mathrm{Hg}$. Optic fundi were normal.

Serum creatinine concentration was $258 \mu \mathrm{mol} / \mathrm{l}$ and serum electrolytes were normal. Urine volume was roughly 2 litres over $24 \mathrm{~h}$. Intravenous urogram (IVU) demonstrated gross bilateral hydroenephrosis with hydro-ureters. Renography showed reduced uptake bilaterally, with an extended time to peak (1050 s) for each kidney and slow excretion suggestive of obstruction. Cystoscopy revealed a normal urethra and a heavily trabeculated bladder containing numerous

(C) The Fellowship of Postgraduate Medicine, 1985 
diverticulae. The prostrate gland was small, and the bladder neck high.

Blood pressure was brought under control gradually by oral labetalol. Trans-urethral resection was then performed and $12 \mathrm{~g}$ prostrate tissue removed. Histology disclosed benign prostatic hyperplasia. Urine volume rose in the post-operative period to over 3 litres per $24 \mathrm{~h}$, and the blood pressure fell to $120 /$ $80 \mathrm{~mm} \mathrm{Hg}$. Labetalol was discontinued after 4 days. Urine volume returned to normal within 10 days. The blood pressure was followed regularly over the next year and the level remained consistently at 120/ $80 \mathrm{~mm} \mathrm{Hg}$. Renography two months after operation showed bilateral normal uptakes and much improved excretory pattern. Sequential changes in GFR and ERPF were as shown (Figure 1).

\section{Case 2}

A male of 63 years had been found 2 years ago to have a normal blood pressure but one month before admission it was found to be $230 / 130 \mathrm{~mm} \mathrm{Hg}$ at a routine check-up. Labetalol was commenced but was not tolerated because of cold extremities. Chlorthalidone plus indapamide was started, but blood pressure control remained poor, necessitating hospital admission.

Nocturia had been present for several months, and urine was voided two or three times each night. There was no frequency by day or hesitancy. Optic fundi were normal. Moderate prostatic enlargement was present. Serum creatinine concentration was $424 \mu \mathrm{mol} / \mathrm{l}$. IVU displayed gross bilateral hydronephrosis with hydro-ureters. Renography demonstrated poor uptake in both kidneys and bilateral obstruction with no response to $10 \mathrm{mg}$ frusemide injection. Cysoscopy revealed a normal urethra with a long prostrate gland. The bladder was heavily trabeculated.

The blood pressure was controlled with triple hypotensive drug therapy: acebutalol $400 \mathrm{mg}$ per day, minoxidil $30 \mathrm{mg} / \mathrm{d}$ and frusemide $40 \mathrm{mg} / \mathrm{d}$, and the level fell from $230 / 130$ to $150 / 95 \mathrm{~mm} \mathrm{Hg}$. Bladder catheterization was accompanied by an increase in urine volume to $3.51 / 24 \mathrm{~h}$ which was associated with a further fall of blood pressure to $130 / 80 \mathrm{~mm} \mathrm{Hg}$. Hypotensive drug therapy was discontinued. Urine volumes returned to normal after one week.

Trans-urethral resection was performed and $85 \mathrm{~g}$ of prostatic tissue removed. Histology disclosed benign prostatic hyperplasia.

The blood pressure remained at $130 / 80 \mathrm{~mm} \mathrm{Hg}$ for two months and then gradually rose to $180 /$ $110 \mathrm{~mm} \mathrm{Hg}$, necessitating re-introduction of the previous triple therapy. Renography two months after operation showed improved uptakes but with still extended times to the renogram peaks $(>900 \mathrm{~s})$. After

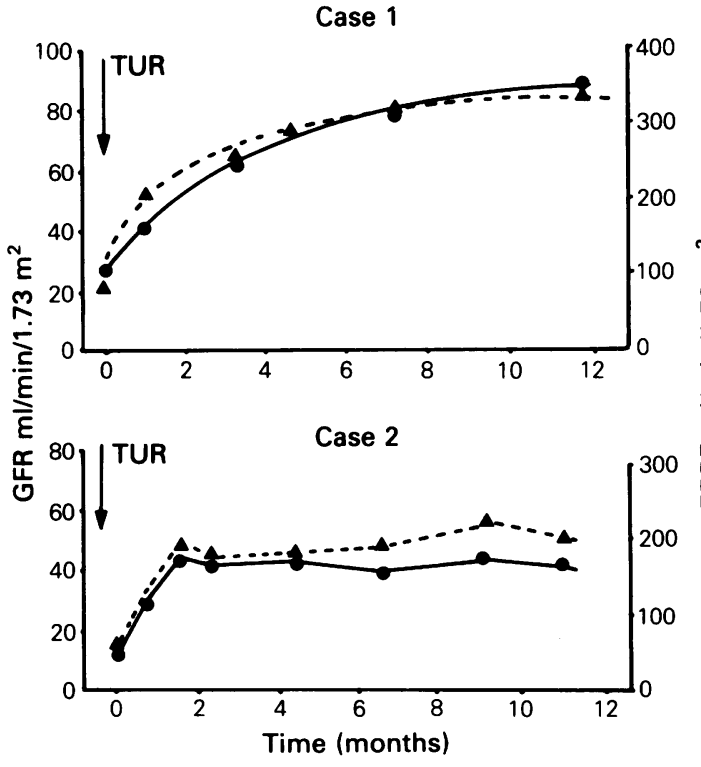

Figure 1 In Case 1 and Case 2, transurethral resection (TUR) was followed by a sequential increase in (O) GFR and $(\Delta)$ ERPF. This effect was blunted in Case 2.

a further two months uptake in the right kidne or became normal but in the left was still reduced. Tim to peak for both renogram curves was near normal. This pattern has persisted. Sequential GFR and ERPF values are shown in the figure (Figure 1).

\section{Discussion}

HPCR should be considered in any middle-aged or elderly male with hypertension of recent onset of any grade of severity. Recognition requires urological assessment, including IVU or renography. Treatment is essentially surgical, and medical treatment with 3 . hypotensive drugs may be counter-productive leading 8 to control of blood pressure in the face of a progressive $₹$ decline in renal function. In the present cases a sharp 0 fall in blood pressure, into the normal range, occurred with relief of lower urinary tract obstruction. In the study of George et al. (1983) in which 14 out of 21 patients were hypertensive, it was observed that $\sigma$ normalization of blood pressure occurred after blad- N der catheterization. However, in the present cases it N was apparent that normalization of blood pressure, $\sigma$ which occurred promptly, was dissociated in time from the slow and gradual recovery of GFR and ERPF, with a return to normal renography pattern, $\stackrel{\oplus}{?}$ which occurred over several months. Recurrence of hypertension, requiring hypotensive drug treatment 
was noted after a few months in Case 2, which was characterized by a lower degree of recovery of GFR and ERPF. Persisting renal damage may have contributed to this resurgence of hypertension.

The aetiology of hypertension associated with bilateral ureteric obstruction and hydronephrosis is poorly understood.

Hypertension accompanying unilateral urinary tract obstruction may be associated with increased renal vein renin levels in the obstructed kidney, and relief of obstruction has been observed to cause a decline of renal vein renin to normal, with normalization of blood pressure (Belman et al., 1968). Weidman et al. (1977) studied six patients before and after surgical treatment for unilateral ureteric obstruction, and noted a correlation between the post-operative blood pressure decline and a fall in plasma renin levels. Vaughan et al. (1974), however, questioned the role of renin secretion in hypertension with unilateral obstruction, as normal renin secretion was demonstrated.

\section{References}

ABRAMS, P.H., DUNN, M. \& GEORGE, N.J.R. (1978). Urodynamic findings in chronic retention and their relevance to results of surgery. British Medical Journal, 2, 1258.

AMUASI, J.H., CRAWLEY, J.C.W., VEALL, N., WILKINS, H.A. \& CRONQUIST, A.G. (1983). Estimation of mean transit times from semi-quantitative indices derived from standard renograms. Clinical Physics and Physiological Measurement, 4, 211.

BELMAN, A.B., KROPP, K.A. \& SIMON, N.M. (1968). Renalpressor hypertension secondary to unilateral hydronephrosis. New England Journal of Medicine, 278, 1133.

GEORGE, N.J.R., O'REILLY, P.H., BARNARD, R.J. \& BLACKLOCK, N.J. (1983). High pressure chronic retention. British Medical Journal, i, 1790.
Bilateral obstruction of the urinary tract may lead to acute-on-chronic renal failure, and associated hypertension may be due to renal parenchymatous disease. The underlying mechanisms are ill-defined, but inappropriately high levels of renin may occur relative to the sodium status of the individual. This may have applied in Case 2 when hypertension recurred some months after TUR. The rapid regression of hypertension in both cases immediately following TUR suggested that post-obstructive diuresis may have ameliorated the hypertensive state, implying that volume expansion from preceding sodium retention may have been aetiologically important.

\section{Acknowledgements}

We are grateful to Mr M.B. Rose for his expert assessment and surgical skills, and to Miss Julie E. Davies for secretarial assistance.

MORGAN, W.D., BIRKS, J.L., SIVYER, A. \& GHOSE, R.R (1977). An efficient technique for the simultaneous estimation of GFR and ERPF, involving a single injection and two blood samples. International Journal of Nuclear Medicine and Biology, iv, 79.

VAUGHAN, E.D. Jr, BUCHLER, F.R. \& LARAGH, J.H. (1974). Normal renin secretion in hypertensive patients with primarily unilateral chronic hydronephrosis. Journal of Urology, 112, 153.

WEIDMANN, P., BERETTA-PICCOLI, C., HIRSCH, D., REUBI, F.C. \& MASSRY, S.G. (1977). Curable hypertension with unilateral hydronephrosis: studies on the role of circulating renin. Annals of Internal Medicine, 87, 437. 